\title{
1 GuideMaker: Software to design CRISPR-Cas guide RNA pools in non-model genomes
}

2 Ravin Poude ${ }^{1,2}$, Lidimarie Trujillo Rodriguez ${ }^{2}$, Christopher R. Reisch ${ }^{2}$, and Adam R. Rivers ${ }^{*}$

$3{ }^{1}$ Genomics and Bioinformatics Research Unit, USDA Agricultural Research Service, Gainesville, FL, 32608,

$4 \quad$ USA

$5 \quad{ }^{2}$ Department of Microbiology and Cell Science, Institute of Food and Agricultural Sciences, University of

6 Florida, Gainesville, FL 326011, USA

$7 \quad *$ Correspondence: adam.rivers@usda.gov

9 Abstract

Background:

11 CRISPR-Cas systems have expanded the possibilities for gene editing in bacteria and eukaryotes. There are

12 many excellent tools for designing the CRISPR-Cas guide RNAs for model organisms with standard Cas

13 enzymes. GuideMaker is intended as a fast and easy-to-use design tool for atypical projects with 1) non-

14 standard Cas enzymes, 2) non-model organisms, or 3) projects that need to design a panel of guide RNAs

15 (gRNA) for genome-wide screens.

\section{Findings:}

17 GuideMaker can rapidly design gRNAs for gene targets across the genome from a degenerate protospacer

18 adjacent motif (PAM) and a GenBank file. The tool applies Hierarchical Navigable Small World (HNSW)

19 graphs to speed up the comparison of guide RNAs. This allows the user to design gRNAs targeting all genes

20 in a typical bacterial genome in about 1-2 minutes.

\section{Conclusions:}


22 Guidemaker enables the rapid design of genome-wide gRNA for any CRISPR-Cas enzyme in non-model

23 organisms. While GuideMaker is designed with prokaryotic genomes in mind, it can efficiently process

24 smaller eukaryotic genomes as well. GuideMaker is available as command-line software, a stand-alone web

25 application, and a tool in the CyCverse Discovery Environment. All versions are available under a Creative

26 Commons CC0 1.0 Universal Public Domain Dedication.

\section{Introduction}

31 CRISPR-Cas technology enables rapid and efficient genome editing in both prokaryotic and eukaryotic cells

$32[1,2]$. CRISPR-based systems are set apart from other genome editing tools by the ease with which they can

33 be programmed to target specific sequences. Almost any DNA sequence in the cell can be targeted as long as

34 it possesses a compatible protospacer adjacent motif (PAM). The PAM is a conserved sequence that flanks

35 the DNA target site, known as the protospacer, and must be present for target recognition [3]. The target specifying guide-RNA (gRNA) can be supplied as RNA, or encoded in DNA, depending on the organism

37 under investigation. Although CRISPR-Cas is often used to edit single genes in eukaryotes, it is increasingly

38 used for other purposes in prokaryotic and eukaryotic organisms, including non-model organisms [4].

41 as the Staphylococcus aureus Cas9 and the Acidaminococcus Cas12a, are also commonly used [6,7]. Accordingly, the

42 parameters for these enzymes are often included in computational tools to identify CRISPR target sites [8-

43 11]. Cas9 enzymes from other organisms and other Cas-associated proteins that can cleave dsDNA, ssDNA,

44 ssRNA, and insert transposon elements have also been described and have their place in molecular toolkits

45 [12-18]. Each of these enzymes generally possesses its own requirements, such as PAM sequence constraints, 
PAM orientation, and protospacer length. Many of these CRISPR-Cas systems have been repurposed to

47 enable molecular genetics techniques like gene deletions, gene insertions, transcriptional depletion and

48 activation, and translational repression [12,19-22]. Some of these techniques can be scaled to the genome

49 level with chip-synthesized oligonucleotides and pooled approaches to screening [23]. In pooled screens,

50 high-throughput DNA sequencing is used to identify how the pool has changed over time to elucidate genes

51 that affect cells' fitness in specific conditions. Given the diversity of the CRISPR systems and their uses,

52 identifying appropriate target sites is not trivial, especially for the number of targets needed for genome-scale

53 experiments.

Here we introduce GuideMaker, a computational tool to identify target sites and design gRNA

55 sequences that is not limited to any specific CRISPR system or organism. Guidemaker is most useful for a

56 few kinds of CRISPR experiments. The first use case is designing pools of gRNAs for genome-wide

57 screening experiments like Perturb-seq and CRISPR pool [23,24]. GuideMaker is optimized for making the

58 all-versus-all comparisons necessary to design a genome-wide screen and return candidate gRNAs for every

59 gene locus. The tool allows the user to filter targets based on their proximity to features of interest, like the

60 start codon for any coding sequence. The second major use case is for researchers working with non-model

61 organisms. Online gRNA design tools often have a limited number of preselected genomes available for

62 analysis because most methods require PAM site positions to be precomputed. GuideMaker rapidly computes

63 all guide positions on demand so the user can provide a set of GenBank files from any organism for analysis.

64 The third use case is for researchers working with Cas enzymes other than the canonical versions of Cas9,

65 Cas12a (Cpf1), or Cas13 with different PAM and target site requirements. GuideMaker allows the user to

66 specify a custom PAM with variable length, including degenerate nucleotides and allows the PAM to be on

67 either the 3 ' or 5 ' side of the protospacer. These features allow GuideMaker to support any current or future

68 CRISPR-Cas system. Since the determination of which CRISPR-Cas system functions best in any given

69 organism is not predictable, this tool is highly relevant to researchers developing CRISPR tools in new

70 species. In some cases, GuideMaker may not be the best choice. There are mature tools for designing gRNAs

71 in model organisms with common CRISPR-Cas systems and targeting a small number of loci [25,26]. Some 
72 of these employ sophisticated statistical models to select the best Cas9 gRNA candidates and may be a better

73 choice for well-studied systems [8]. Because the re is limited experimental data on most Cas/organism

74 combinations, GuideMaker relies on design heuristics rather than machine learning-based identification

75 methods.

76

\section{Methods}

Main features, input parameters, and workflow

79 GuideMaker is designed to be easy to use as either a web application or a command-line utility. The key

80 features of GuideMaker are:

81 1. All the potential guides in a genome can be quickly designed in one run.

82 2. It can design gRNAs for any small to medium size genome (up to about 500 megabases).

3. It can design gRNAs for any PAM sequence from any Cas system.

4. Search is customizable through user-defined guide parameters (as highlighted in Figure 1). These features are specific to organisms, CRISPR-Cas systems, and experiments. Tuning these parameters can improve the sensitivity and specificity of gRNA.

5. Users can exclude specific restriction sites from guides to preserve those sites for downstream experiments. realistic control gRNAs that are highly divergent from sequences adjacent to PAM sites.

927 Provides an interactive visualization and exploratory tool to evaluate the guides.

93 8. Provides tabular result files which can be used for the design and ordering of gRNAs.

949 9. The software can be run as a web application [27], a CyVerse application, or a command-line 95 application [28]. Server code is included for running local instances of the web application as well. 
97 uploads the input genome in one or more gbk or gzipped .gbk files and defines the PAM and gRNA

98 parameters (as highlighted in Figure 1). Guidemaker identifies and filters target sites, then returns summary

99 data to the graphical environment (Figure 2). Users can use the interactive plots to learn more about the on accessing the software through various methods is available on the project homepage [32].

\section{Search method}

$$
\text { GuideMaker initially scans the genome, recording all candidate guide sequences adjacent to the }
$$
specified PAM sequence on both DNA strands (Figure 3). Candidate guides are then optionally checked for

111 the restriction sites. Next, the candidates guides are searched for a unique "seed region" closest to the PAM

112 site and candidate gRNAs that are not unique in their "seed region" are removed. Then, approximate nearest

113 neighbor search is used to remove candidate guides too similar to PAM adjacent sequences in the genome,

114 based on Hamming distance (the number of substitutions required to turn one DNA sequence into another

115 equal-length sequence). The approximate nearest neighbor search is performed using the Hierarchical

116 Navigable Small World (HNSW) graph method in the Non-Metric Space Library (NMSLIB) [33,34]. An

117 index of all the initial candidate guides is created using the bitwise Hamming distance metric. Each guide with

118 a unique "seed region" is compared to all candidate guides and any guides with Hamming distances below the

119 user-set threshold are removed. This differs from the standard procedure of indexing the genome and

120 mapping each candidate guide against the whole genome then parsing each result. HNSW has a search 
121 complexity of $\mathcal{O}(\log N)$ and index complexity of $\mathcal{O}(N \cdot \log N)$ [33]. Finally, user-defined criteria are applied

122 specifying the proximity and orientation of guides relative to genomic features like genes. A list of guides is

123 then returned to the user with relevant information about the guide and its target genomic features. script for re-optimization (flag --config) of these hyper-parameters is included in the command-line version of the software.

\section{Computational performance} scalability of GuideMaker (Supplementary Table 1). For benchmarking the performance, the same parameters were used unless a specific parameter was being tested: a PAM motif of 'NGG', 3' pam orientation, target length of 20, 1sr (length of seed region) of 11, before and after parameters of 500, knum of 10, controls of 10 ,

137 dist of 3 and threads of 32 . We profiled the performance of GuideMaker with different threads [1, 2, 4, 8, 16, 138 and 32] in processors with and without the AVX2 processor instruction set. All tests were run on a single

139 compute node with 2 x 24 core Intel(R) Xeon(R) Platinum 8260 CPU @ 2.40 GHz with Cascade Lake

140 microarchitecture. Three bacterial genomes, a fungal genome, and a plant genome were used in performance

141 benchmarking: Escherichia coli (K12), Pseudomonas aemuginosa (PAO1), Burkholderia thailandensis (E264), Arabidopsis

142 thaliana, and Aspergillus fumigatus. For the gene or locus-specific comparisons, only the guides within the locus

143 coordinates (i.e. zero feature distance) were considered.

\section{Comparison to existing design method}




\section{Results}

The time for Guidemaker to complete a typical run identifying all SpCas9 gRNAs (PAM 'NGG') in a bacterial genome using 8 compute cores was 75 seconds for E. coli and 130 seconds for P. aeruginosa (Figure 4). For SaCas9 and StCas9, which have a longer PAM sequence (NGRRT' and 'NNAGAAW' respectively, with 3' PAM orientation) and the reby fewer potential targets, the same genomes ran in 19 or 5 seconds

163 (Supplementary Figures 1). The fungus Aspergillus fumigatus (28MB) and plant Arabidopsis thaliana (114 MB)

164 have larger genomes but are still processed quickly. A. fumigatus processed between $23-304$ seconds, while

167 (AVX2) on newer $\mathrm{x} 86$ processors, which improves the search speed because HNSW search is accelerated with AVX2 (Supplementary Figure 3). The acceleration was larger when fewer processors were available 
171 so the software scales well when additional cores are added up to 8 cores (Supplementary Figure 3). In

172 practice it scaled up sub-linearly with genome size, globally estimating Cas9 guides for E. coli MG1655

173 (4.6MB) in 75 seconds and A. thaliana (114.1MB) in 921 seconds, both on 8 cores (Memory usage: 1.9GB for

174 E. coli and $15.4 \mathrm{~GB}$ for A. thaliana, Supplementary Figure 4).

175 The results of Guidemaker were compared with the popular guide design software CHOPCHOP

176 version 3 [35]. When GuideMaker's filtering settings are set to match CHOPCHOP, the results are very

177 similar and $99.9 \%$ of the targets identified by GuideMaker fall within 2 bp of target coordinates returned by

178 CHOPCHOP. When GuideMaker's unique seed region criterion was not applied at the loci level, the average

179 number of guides identified by the two approaches was similar per locus (Mean GuideMaker $=116.8$, Mean

180 CHOPCHOP $=113.6$, p-value $=0.86$, Supplementary Table 2). Although the number of guides identified

181 per gene locus differed, none of the genes were missed by either tool. GuideMaker's default requirement of a

182 seed region is more stringent than CHOPCHOP, and with it enabled, GuideMaker returns (count=1787)

$18338.4 \%$ (for $2 \mathrm{Kbp}-42 \mathrm{Kbp}$ regions) of the targets compared to CHOPCHOP (count=4651) E. coli K12. At the

184 sequence level, $96.7 \%$ of the identified gRNA (1729/1787) from both of the tools had identical sequences.

185 The more stringent filtering could potentially reduce off-targeting but that would need to be experimentally

186 validated in a range of organisms. The ratio of gRNA found by both the tools across the multiple $40 \mathrm{Kbp}$

187 regions was 39.2\% (sd= 1.9\%, Supplementary Table 3) when using Guidemaker's more stringent default

188 settings. This ratio was calculated by dividing the number of gRNA from GuideMaker by the number from

189 CHOPCHOP for each $40 \mathrm{~Kb}$ region.

191 Discussion

192 Designing gRNAs is a two-step process where GuideMaker first identifies potential guides adjacent to PAM 193 sequences and then filters the potential guides based on multiple criteria. The most important criterion is that 194 each guide has a minimum edit distance from any other sequence adjacent to a PAM site in the genome; this 195 decreases the likelihood of off-target binding. The second way GuideMaker reduces off-target binding is by 
requiring that a set number of bases near the PAM site are unique from any other candidate guide. The 8

197 bases nearest the PAM are the most important for target specificity, and any mismatch is sufficient to prevent

198 binding $[36,37]$. The length of the unique region should be set with consideration for the size of the genome

199 since requiring short unique regions will limit the number of total guides that can be found. For example,

200 requiring that every gRNA be unique in the first 3 bp would only allow for $4^{3}=64$ possible guides to be

201 designed. For normal --lsr values of 9-12 this is only limiting for human-sized genomes and can be disabled by

202 setting --lsr to 0. All guides designed by GuideMaker are perfect matches to a single site in the genome.

203 Specificity is obtained by requiring all similar PAM-adjacent sequences to be unique in the critical "seed

204 region" and have a total number of mismatches that exceed the user-defined threshold. This double criterion

205 is expected to increase specificity.

The primary goal of the current version of our software is to support the design of gRNAs in non-

standard Cas enzymes for non-model organisms at the genome-scale. It is known that gRNA's do not

208 perform equally, thus empirical experiments will be needed to fully validate the functionality and efficacy of

209 gRNA predictions. Given the similarity in targets identified by GuideMaker and CHOPCHOP, we anticipate

210 performance will be similar to the current state of the art but applicable to more design use cases. When a

211 unique seed region and Hamming distance-based filters were applied, GuideMaker created guides more

212 conservatively, generating only about $40 \%$ of the guides created by CHOPCHOP. While CHOPCHOP has

213 an option to specify the maximum number of mismatches in the first $9 \mathrm{bp}$ or the whole guide, it does not

214 allow the application of both criteria. While there are small differences in the number and position of guides

215 generated by GuideMaker, with GuideMaker being more conservative by default, both programs create

216 enough guides to target nearly all gene loci in the genome of E. coli. If experimentally validated data become

217 available from genome-wide screens with different Cas enzymes, the future versions of GuideMaker could

218 potentally incorporate scoring matricies to help rank candidate guides. 
221 index and search new genomes. Several parameters can be tuned to ensure compatibility with the specific

222 application of the user. For example, GuideMaker checks the designed gRNA for a given restriction enzyme

223 site to prevent incompatibility with the cloning strategy. Second, the maximum distance from a target

224 sequence from the start of an annotated feature can be chosen to disrupt promoters or the beginning of the

225 coding sequence, since these sites are preferred for CRISPRi experiments. GuideMaker also creates off-target

226 gRNAs for use as negative controls in high-throughput experiments. Lastly, the program plots the results for

227 visual exploration of the targets and exports the data as .csv files. The software is available as a command-line

228 application, a web application, and is integrated into the CyVerse Discovery Environment to provide users

229 with a range of usage options.

\section{Availability and Requirements}

232 Project name: GuideMaker

233 Project home page: https://guidemaker.org

234 Operating system(s): Linux or MacOS

235 Programming language: Python $>=3.6$

236 Other requirements: 'pybedtools $==0.8 .2$ ', 'nmslib $>=2.0 .6$ ','altair', 'streamlit $>0.80 .0$

237 License: CC0 1.0 Public Domain Dedication

\section{Competing Interests}

241 Authors declare no competing interests 


\section{Data Availability}

244 The source code and command-line executables for GuideMaker are available at the Zenodo [38] and can be

245 installed directly from Github [28], Bioconda [29], or as a Docker container [30]. Data and code to reproduce

246 the analysis in the paper are available at Zenodo [39]. As a work of the United States Department of

247 Agriculture, Guidemaker is released to the public domain under a Creative Commons (CCO) public domain

248 attribution. The program is also available as a web application through the Cyverse discovery environment

249 [31], and as a stand-alone web application [27].

251 Additional Files

252 Supplementary Figure 1. Performance of GuideMaker for SaCas9 and StCas9.

253 Supplementary Figure 2. Performance of GuideMaker for SpCas9, SaCas9, and StCas9.

254 Supplementary Figure 3. Performance of GuideMaker with AVX2 settings.

255 Supplementary Figure 4. Memory usage of GuideMaker for SpCas9, SaCas9, and StCas9.

256 Supplementary Table 1: Organism features

257 Supplementary Table 2: Comparison of the average number of gRNA identified by GuideMaker and

258 CHOPCHOP.

259 Supplementary Table 3: Comparison of consensus ratio between GuideMaker and CHOPCHOP.

\section{List of abbreviations}


262 CAS: CRISPR-associated protein; CRISPR: Clustered Regularly Interspaced Short Palindromic Repeats;

263 gRNA: Guide RNA; HMSW: Hierarchical Navigable Small World; NMSLIB: Non-Metric Space Library;

264 PAM: Protospacer Adjacent Motif

\section{Funding}

267 The research was supported by the United States Department of Agriculture (USDA), Agricultural Research

268 Service (ARS) project number 6066-21310-005-D, and ARS cooperative agreement 6066-21310-005-28-S to

269 the University of Florida. This research used resources provided by the SCINet scientific computing initiative 270 of the USDA-ARS, ARS project number 0500-00093-001-00-D.

\section{Author Contributions}

273 R.P., L.T.R., C.R.R., and A.R.R. conceived and designed the study. R.P. and A.R.R developed and optimized 274 the software and performed the experiments. R.P., L.T.R., C.R.R., and A.R.R, tested the software, wrote, and 275 revised the manuscripts. All authors read and approved the final manuscript.

277 References

278 1. Jiang W, Bikard D, Cox D, Zhang F, Marraffini LA. RNA-guided editing of bacterial genomes using

279 CRISPR-Cas systems. Nat Biotechnol. 2013; doi: 10.1038/nbt.2508.

280 2. Ran FA, Hsu PD, Wright J, Agarwala V, Scott DA, Zhang F. Genome engineering using the CRISPR-Cas9

281 system. Nat Protoc. 2013; doi: 10.1038/nprot.2013.143.

282 3. Mojica FJM, Díez-Villaseñor C, García-Martínez J, Almendros C. Short motif sequences determine the

283 targets of the prokaryotic CRISPR defence system. Microbiology. 2009; doi: 10.1099/mic.0.023960-0. 
284 4. Pickar-Oliver A, Gersbach CA. The next generation of CRISPR-Cas technologies and applications. Nat

285 Rev Mol Cell Biol. 2019; doi: 10.1038/s41580-019-0131-5.

286 5. Deltcheva E, Chylinski K, Sharma CM, Gonzales K, Chao Y, Pirzada ZA, et al.. CRISPR RNA maturation

287 by trans-encoded small RNA and host factor RNase III. Nature. 2011; doi: 10.1038/nature09886. Staphylococcus aureus Cas9. Nature. 2015; doi: 10.1038/nature14299. single RNA-guided endonuclease of a class 2 CRISPR-Cas system. Cell. 2015; doi: 10.1016/j.cell.2015.09.038. to maximize activity and minimize off-target effects of CRISPR-Cas9. Nat Biotechnol. 2016; doi: $10.1038 /$ nbt.3437. editing efficiency. Luigi Martelli P, editor. Bioinformatics. 2020; doi: 10.1093/bioinformatics/btaa041. sgRNA design. Genome Res. 2015; doi: 10.1101/gr.191452.115. improved single and paired CRISPR guide RNA design. Nat Biotechnol. 2017; doi: 10.1038/nbt.3804.

12. Anzalone A V., Koblan LW, Liu DR. Genome editing with CRISPR-Cas nucleases, base editors, transposases and prime editors. Nat Biotechnol. 2020; doi: 10.1038/s41587-020-0561-9. 
14. Abudayyeh OO, Gootenberg JS, Konermann S, Joung J, Slaymaker IM, Cox DBT, et al.. C2c2 is a single-

15. Ma E, Harrington LB, O'Connell MR, Zhou K, Doudna JA. Single-stranded DNA cleavage by divergent CRISPR-Cas9 enzymes. Mol Cell. 2015; doi: 10.1016/j.molcel.2015.10.030.

RNA-guided DNA integration. Nature. 2019; doi: 10.1038/s41586-019-1323-z. insertion with CRISPR-associated transposases. Science. 2019; doi: 10.1126/science.aax9181. 
326 23. Dixit A, Parnas O, Li B, Chen J, Fulco CP, Jerby-Arnon L, et al.. Perturb-Seq: Dissecting molecular

327 circuits with scalable single-cell RNA profiling of pooled genetic screens. Cell. 2016; doi:

$328 \quad 10.1016 /$ j.cell.2016.11.038.

329 24. Peters JM, Colavin A, Shi H, Czarny TL, Larson MH, Wong S, et al.. A comprehensive, CRISPR-based

330 functional analysis of essential genes in bacteria. Cell. 2016; doi: 10.1016/j.cell.2016.05.003.

331 25. Zhu LJ. Overview of guide RNA design tools for CRISPR-Cas9 genome editing technology. Front Biol.

332 2015; doi: 10.1007/s11515-015-1366-y.

333 26. Cui Y, Xu J, Cheng M, Liao X, Peng S. Review of CRISPR/Cas9 sgRNA design tools. Interdiscip Sci

334 Comput Life Sci. 2018; doi: 10.1007/s12539-018-0298-z.

27. GuideMaker. The GuideMaker web app. https://guidemaker.app.scinet.usda.gov. Accessed 2021 May 27.

28. GuideMaker 2021. GuideMaker (Version 0.2.0). https://github.com/USDA-ARS-

29. GuideMaker. The GuideMaker bioconda installation. https://anaconda.org/bioconda/guidemaker.

GBRU/packages?repo_name=GuideMaker.

341 31. Merchant N, Lyons E, Goff S, Vaughn M, Ware D, Micklos D, et al.. The iPlant collaborative:

342 cyberinfrastructure for enabling data to discovery for the life sciences. PLOS Biol. 2016; doi:

$34310.1371 /$ journal.pbio.1002342.

344 32. GuideMaker. The GuideMaker project homepage. https://guidemaker.org. Accessed 2021 June 18.

345 33. Malkov YA, Yashunin DA. Efficient and robust approximate nearest neighbor search using hierarchical 346 navigable small world graphs. IEEE Trans Pattern Anal Mach Intell. 2020; doi:

347 10.1109/TPAMI.2018.2889473. 
367 CRISPR-Cas effector complexes: diversified mechanisms and structures. RNA Biol. 2019; doi: 
371 43. Wu X, Kriz AJ, Sharp PA. Target specificity of the CRISPR-Cas9 system. Sander JD, Joung JK, editors.

372 Quant Biol. 2014; doi: 10.1007/s40484-014-0030-x.

Figure 2. A typical workflow of GuideMaker: 1) A user uploads the input genome (single or multiple) as

379 Genbank file, then defines the PAM sequence along with all the associated parameters and submits them to

380 run the program. 2) GuideMaker processes the input files and generates the interactive plots. Users can use

381 these interactive plots to explore the results and sort them by locus tag and genome coordinates. 3)

382 GuideMaker provides all the results and log files as downloads under the "Results" section.

Figure 3. Entity Relationship Diagram showing the operation of the GuideMaker core program.

Figure 4. Performance of GuideMaker for SpCas9. Evaluating the performance of GuideMaker across

387 three bacterial genomes using the "NGG" PAM motif with a target length of 20, unique zone of 11, 3prime

388 PAM orientation, before and into parameters of 500 , knum of 10 , controls of 10 , and dist of 3 . The mean of

38910 runs was used for the evaluation, where dot and bar represent the mean and standard error, respectively. 
GuideMaker accepts one or more Genbank (.gbk or gzipped .gbk.gz) files with sequence data from a single genome as an input. GuideMaker extracts all the required information from the Genbank file to identify gRNAs and genomic features, allowing users to globally create gRNAs without preprocessed mapping files. Option: --genbank

The Protospacer Adjacent Motif (PAM) is a short, generally 2-8 bp, sequence essential for binding by the Cas protein[3,40,41]. GuideMaker provides users the flexibility to define the PAM sequence for any Cas protein, enabling usage of new CRISPR-Cas systems. Degenerate PAM sequences are allowed. Option: --pamseq

Restriction GuideMaker allows users to provide a list of defined or degenerate restriction site sequences to exclude from

Enzymes guides, which may be needed for some vector systems. Option: --restriction_enzyme_list.
Carsonella ruddii.gbk.gz Carsonella_ruddii.gbk

NGG (SpCas9)

NGRRT (SaCas9)

GAATTC; Default: None The PAM orientation parameter defines PAM position relative to the protospacer. Depending on the CRISPR-Cas

PAM system, the PAM could be 5' or 3' side of the guide sequence. For instance, SpCas9 recognizes 'NGG' PAM on the $3^{\prime}$ end of the guide (i.e. 5'-[guide][pam]-3'), whereas the Cpf1 PAM is on the $5^{\prime}$ end of the guide sequence (i.e. 5'[pam][guide]-3'). To accommodate such differences, GuideMaker offers flexibility to define the PAM orientation. Option: --pam_orientation.

Orientation Guide length $\begin{aligned} & \text { Guide length defines the length of gRNA. Changing the guide length allows the user to adjust the gRNA efficacy } \\ & \text { and specificity [42]. GuideMaker allows users to select the length of gRNA within 10-27 bp. Option: --guidelength. }\end{aligned}$

The seed region is the guide sequence closest to the PAM recognition site, and the distal region is the region furthest from the PAM. For instance, if the guide length is $22 \mathrm{bp}$, and the length of the seed region is 10 , then the size of the seed and the distal regions is 10 and 12, respectively. It has been shown that the region close to PAM is sensitive [36,43], and non-uniqueness in this region can lead to off-target matches; however, the importance of the seed region is specific to the CRISPR-Cas system and the organism. Thus, GuideMaker allows the user to define the seed region with the maximum length of $27 \mathrm{bp}$; although, the length of the seed region must be less than or equal to the Guidelength. Additionally, the length of the seed region should not be too small because the total number of possible guides is limited to 4 raised to the power of the seed length. Option: --Isr.

seed region

\begin{tabular}{|c|c|c|}
\hline \multicolumn{3}{|c|}{ 3' PAM } \\
\hline $5^{\prime}$ & & PAM \\
\hline \multicolumn{3}{|c|}{$5^{\prime}$ PAM } \\
\hline $5^{\prime}$ & PAM & gRNA \\
\hline & \multicolumn{2}{|c|}{ Guide length } \\
\hline & & PAM \\
\hline
\end{tabular}

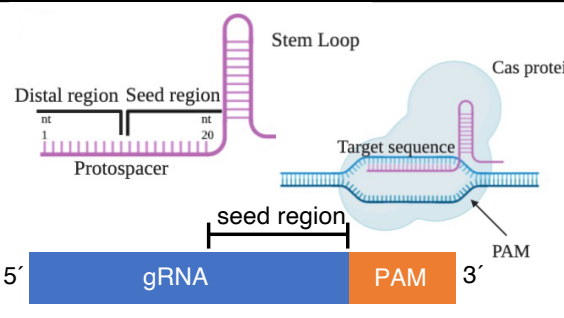

Hemming distance defines the number of substitutions required to turn one DNA sequence into another equal-

length sequence. GuideMaker calculates the Hamming distance between all the candidate gRNAs and all

Distance sequences adjacent to a PAM site. gRNAs with a distance less than or equal to the user-defined value are considered too similar and removed to minimize off-targeting. Option: --dist

$\begin{array}{ll}\text { Before } & \text { Before parameter allows user to select gRNAs that are upstream of a feature's start site. For } \\ \text { set to } 100, \text { each gRNA within } 100 \mathrm{bp} \text { upstream of a feature will be retrieved. Option: --before }\end{array}$

The into parameter allows the user to select gRNAs that are downstream of a feature's start. For example, if "into" is set to 100, each gRNA within 100 bp downstream of a feature will be retrieved. Option: --into.

Similar guides The number of sequences similar to the gRNA to include in the design report. Option: --knum

Options: [ $0-5$ ]; Default: 2

Options: [ $1-500$ ]; Default: 100

Options: [ 1 - 500 ]; Default: 200

Options: [ 2 - 20 ]; Default: 3

Control

gRNAs 
bioRxiv preprint doi: https://doi.org/10.1101/2021.06.28.450164; this version posted June 29, 2021. The copyright holder for this preprint (which was not certified by peer review) is the author/funder. This article is a US Government work. It is not subject to copyright under 17 USC 105 and is also made available for use under a CCO license.

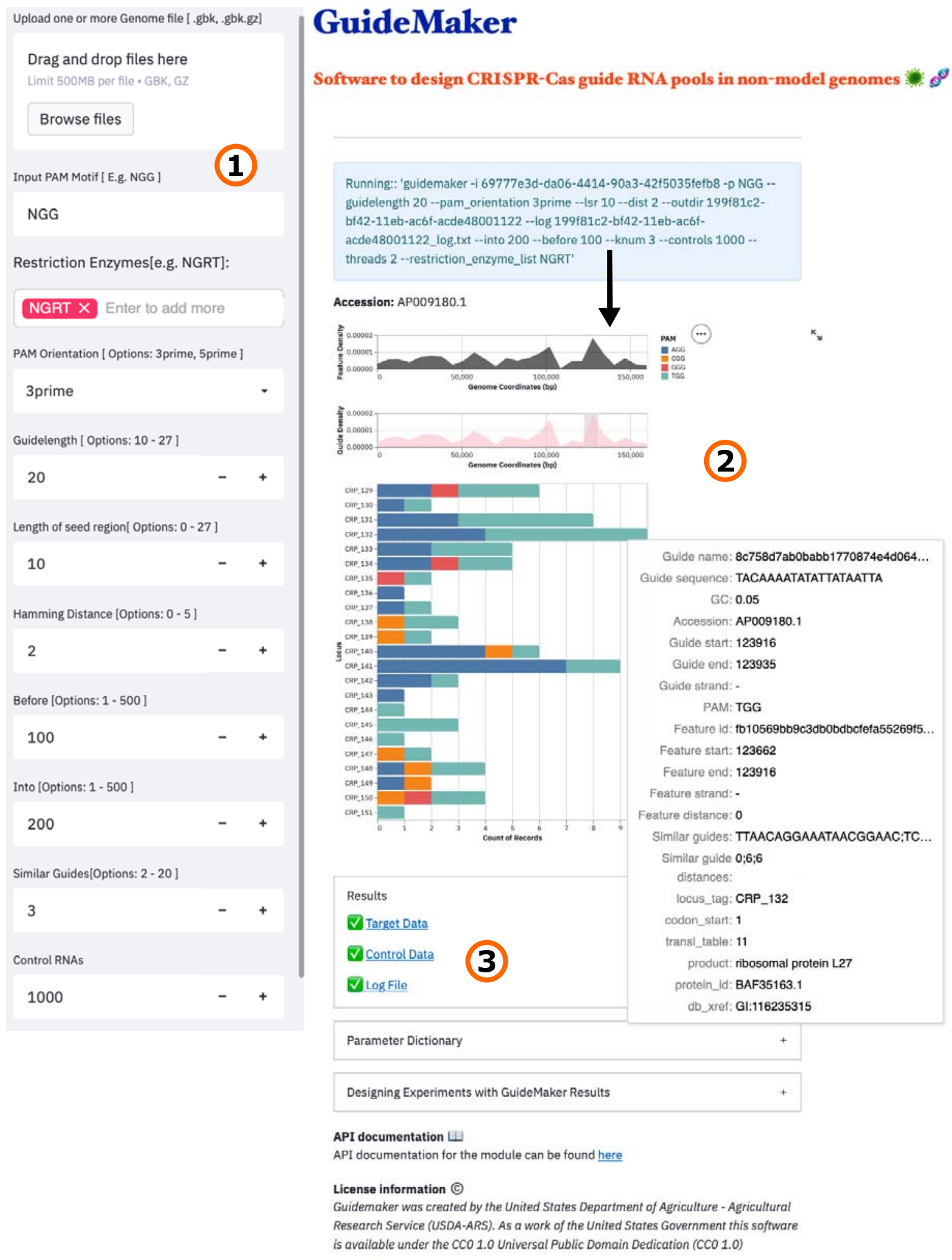


bioRxiv preprint doi: https://doi.org/10.1101/2021.06.28.450164; this version posted June 29, 2021. The copyright holder for this preprint (which was not certified by peer review) is the author/funder. This article is a US Government work. It is not subject to copyright under 17 USC 105 and is also made available for use under a CCO license.

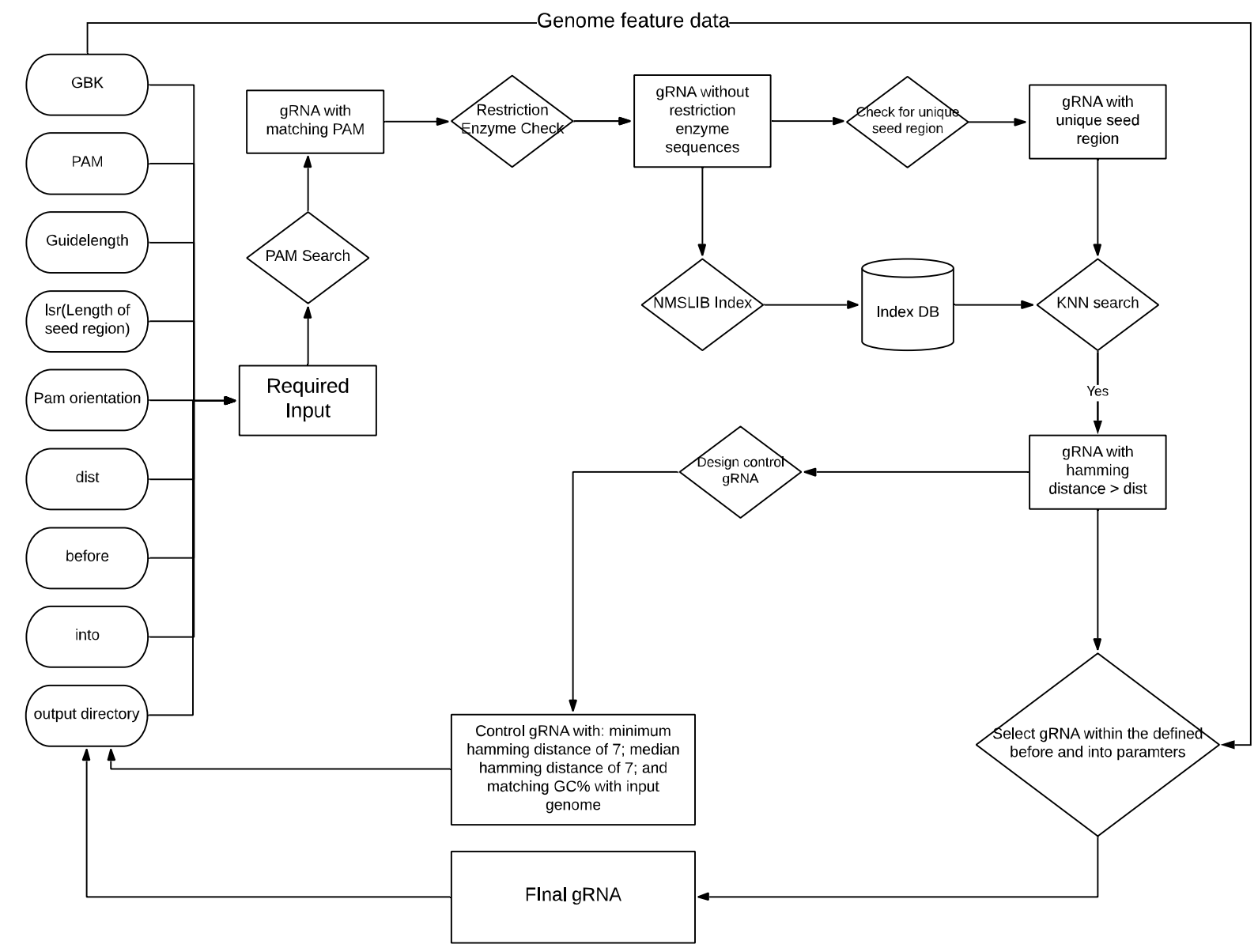


bioRxiv preprint doi: https://doi.org/10.1101/2021.06.28.450164; this version posted June 29,2021 . The copyright holder for this preprint (which was not certified by peer review) is the author/funder. This article is a US Government work. It is not subject to copyright under 17 USC 105 and is also made available for use under a CCO license.

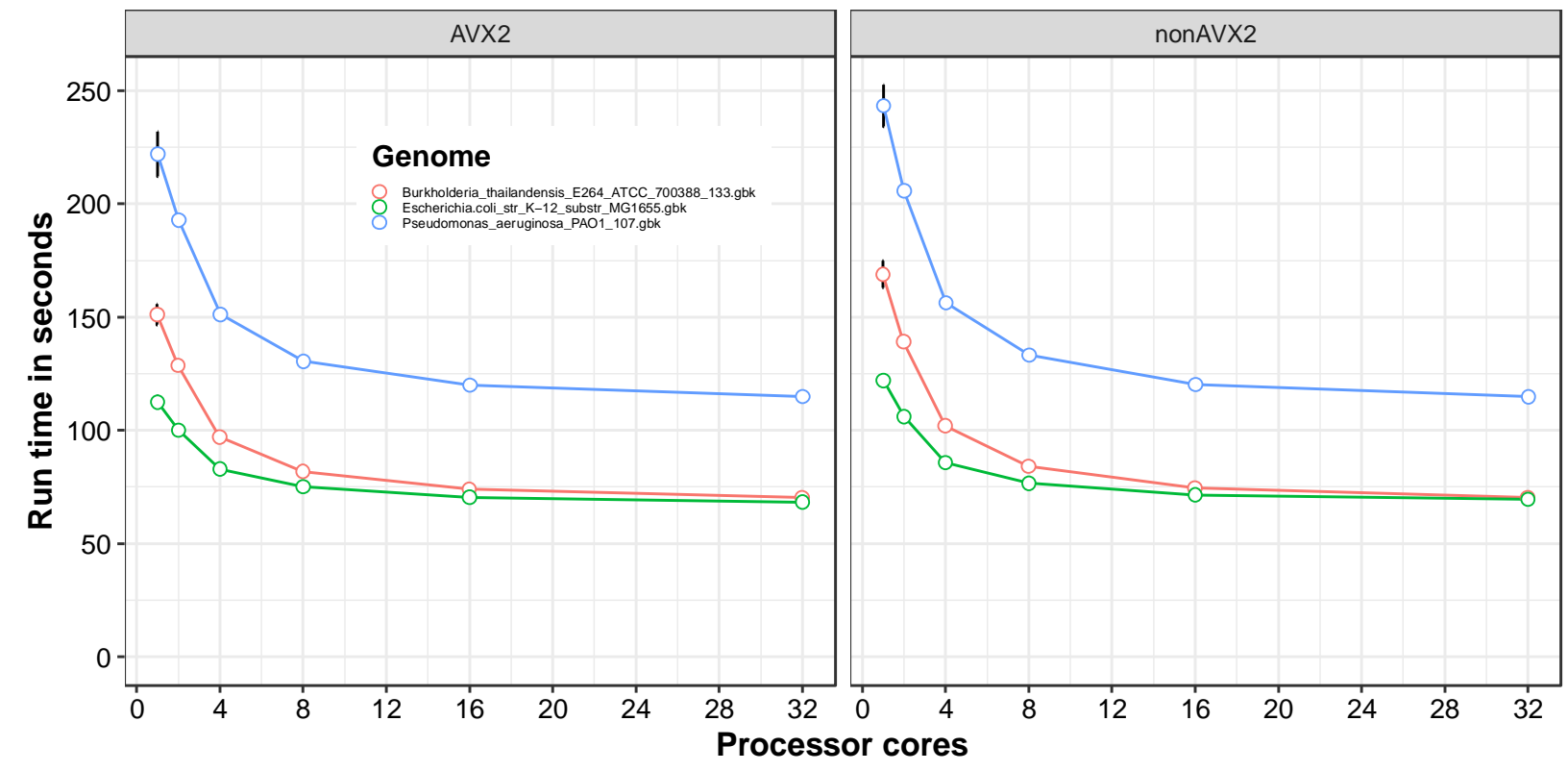

